\title{
Transidentitaire : Sexe, Genre et Sexualité dans Volkswagen Blues de Jacques Poulin et Le Goût des Jeunes Filles de Dany Laferrière
}

\author{
RACHEL BAKER* \\ University of Victoria \\ bakerra@uvic.ca
}

\begin{abstract}
Résumé
À l'heure où les rôles de genre traditionnels sont invariablement réexaminés, déconstruits et réécrits, les conceptions de la masculinité et de la féminité prennent une importance majeure. Cet article examine la multiplicité des identités de genre dans la littérature québécoise contemporaine: Volkswagen Blues de facques Poulin et Le Goût des jeunes filles de Dany Laferrière. À partir d'un modèle transidentitaire basé sur des travaux théoriques d'Isabelle Boisclair et de Lori Saint-Martin, deux critiques littéraires québécoises, on examine la manière dont ces écrivains évoquent la masculinité et la féminité. Poulin et Laferrière abordent les questions du sexe et de l'orientation sexuelle, et celles du genre, de son identité et de son expression, créant des espaces où les corps oscillent souvent entre masculin et féminin et ont des qualités masculines et féminines qui apparaissent simultanément ou en alternance, ainsi rejetant un modèle binaire traditionnel du genre.
\end{abstract}

\footnotetext{
"Ce projet a été soutenu par la bourse Jamie Cassels Undergraduate Research Award. Je remercie chaleureusement Dr. Dave McKercher. Je lui serai toujours gré de son partage des connaissances et son enseignement passionné. De même, je remercie les professeurs au département de français, sans lesquels je n'aurais pensé à faire des études littéraires : Mme. Annye Castonguay, Dr. Marc Lapprand, Dr. Catherine Léger et Dr. Sada Niang. En particulier, je suis reconnaissante à ma directrice de thèse, Dr. Marie Vautier, dont l'expertise et l'amour de la littérature québécoise m'ont guidée jusque-là. Les encouragements reçus le long du projet m'ont été d'une aide précieuse. Je remercie également mon deuxième lecteur, Dr. Emile Fromet de Rosnay, de son appui et de ses judicieux conseils. Et bien sûr, je remercie mes diverses communautés de leur soutien indéfectible et de leur diversité précieuse.
} 
À travers les devises narratives, les textes présentent des personnages qui occupent le continuum du genre et effectuent leurs identités en fonctions des contextes dans lesquels ils se trouvent. Enfin, ces oeuvres encouragent le lecteur de s'interroger sur leur conception du genre, ce que veut dire être homme et ce que veut dire être femme.

Keywords : transidentitaire; masculinité ; féminité ; genre ; sexualité ; identité sexuelle ; multiplicité de genre ; genderqueer ; études littéraires du genre; littérature contemporaine québécoise; Dany Laferrière; Le Goût des jeunes filles; Jacques Poulin; Volkswagen Blues; continuum du genre

\section{INTRODUCTION}

L'écriture est aussi un espace pour rêver, et pour croire qu'il est possible d'être né à Port-au-Prince, de vivre à Montréal et de penser qu'on est un écrivain japonais.

- Dany Laferrière

$\mathrm{E}$

N PLUS D'ÊTRE UN ESPACE POUR RÊVER, la littérature est un lieu transformateur, et ainsi elle joue un rôle essentiel dans le dialogue qui se déroule autour du genre, autant à l'intérieur qu'à l'extérieur des frontières littéraires et du cadre universitaire. Les théories genderqueer et les études du genre (Gender Studies) sont de plus en plus au premier plan dans de nombreux domaines. Les études littéraires trans ${ }^{*}$ 团 peuvent sensibiliser les lecteurs à l'évolution des

1. GATE (Global Action for Trans* Equality - http ://transactivists.org/trans/) utilise le terme trans* pour décrire les individus qui transgressent les normes (binaires) (occidentaux) du genre. Trans ${ }^{*}$ comprend ceux qui ont une identité de genre qui n'est pas le genre assigné à la naissance et/ou ceux qui se sentent obligés, qui préfèrent ou qui choisissent - soit par des vêtements, des accessoires, des produits cosmétiques ou des modifications du corps - de se présenter autrement qu'aux attentes du rôle assigné à leur sexe de naissance. Cela comprend entre autres les individus transsexuels et transgenres, les travestis, les « cross-dressers », les individus sans genre et genderqueer. Le terme devrait être vu comme un indicateur de plusieurs identités, la plupart desquelles sont particulières à des cultures locales et à des contextes historiques, décrivant des individus qui élargissent et diversifient la conception binaire du genre (ma traduction). 
conceptions du sexe, du genre et de la sexualité dans l'espoir que l'exposition continue engendrera davantage d'empathie pour le nombre croissant d'individus qui ne relèvent pas du champ identitaire binaire. Volkswagen Blues (1984) de Jacques Poulin et Le Goût des jeunes filles [1992] (2004) de Dany Laferrière s'éloignent de cette binarité et participent à ce dialogue autour du genre, ce qui justifie de les étudier. Ces textes nous invitent à nous poser une question toute simple :

"Qu'est-ce qu'un homme? Qu'est qu'une femme? » La réponse, elle, n'est pas binaire.

Ce travail, divisé en quatre parties, explore les interactions entre la masculinité et la féminité, et la façon dont les écrivains créent des espaces où les corps de papier subvertissent la stricte binarité traditionnelle de ces catégories. La première partie décrit le modèle « postmoderne » de la conception du genre développé par Isabelle Boisclair et Lori Saint-Martin (2006), et présente les écrivains et les romans; la deuxième partie porte sur le sexe des personnages et analyse si leurs performances des rôles sociaux entrent en conflit avec les attentes traditionnelles du sexe biologique; la troisième partie examine le genre des personnages et la manière dont les textes évoquent le masculin et le féminin; et l'étude de la vision de la sexualité véhiculée par les textes constitue la quatrième partie. Laferrière et Poulin créent des espaces littéraires où les personnages glissent du masculin au féminin à travers une multiplicité de perspectives, et où les traits genrés ne correspondent pas toujours avec le sexe biologique. L'analyse compare et contraste les textes pour mieux comprendre les enjeux et les procédés qui, malgré la mise en scène de couples mâle/femelle, véhiculent une conception que je qualifierai de «transidentitaire » du sexe, du genre et de la sexualité.

\section{UN MODÈLE « POSTMODERNE 》 DES IDENTITÉS SEXUELLES DANS LA LITTÉRATURE QUÉBÉCOISE CONTEMPORAINE}

Dans l'article, "Les conceptions de l'identité sexuelle, le postmodernisme et les textes littéraires », Isabelle Boisclair et Lori Saint- 
Martin (2006) proposent trois modèles pour concevoir l'identité sexuelle dans le but de montrer comment certains écrivains véhiculent une conception du sexe, du genre et de la sexualité qui bouleverse le système identitaire traditionnel. Le premier modèle, surnommé " patriarcal », soutient que les hommes et les femmes existent dans un système de genre binaire, où l'identité de genre (homme/femme) est conforme au sexe biologique (mâle/femelle). À l'intérieur de ce système phallocentrique, adopter le comportement du genre opposé est contre nature et l'hétérosexualité est la norme obligatoire; toutes les autres expressions de sexualité sont aberrantes. Le deuxième modèle, surnommé " féministe », privilégie l'égalité entre les sexes et se manifeste lors de l'émergence du féminisme dans les années 1970. En plus de l'égalité, il valorise l'homosexualité, ainsi rejetant la présomption d'hétérosexualité. Pour ce qui est du sexe, le modèle est toujours binaire. C'est-à-dire, l'identité de genre et/ou le comportement du genre peuvent ne pas correspondre au sexe biologique, mais les pôles eux-mêmes (homme/femme) ne sont pas remis en cause.

Le troisième modèle pour concevoir l'identité sexuelle, que Boisclair et Saint-Martin surnomment "postmoderne », décloisonne la binarité traditionnelle du sexe, du genre et de la sexualité, et coïncide avec le décentrement de la littérature québécoise dès les années 1980, où celle-ci « entre dans l'ère du pluralisme » (Biron, Dumont et Nardout-Lafarge, 2010, p. 531). Désormais, la question identitaire fait éclater le paysage littéraire. Selon Sherry Simon (1991), l'identitaire renvoie à l'identité considérée comme une construction et, dans le contexte québécois, celle-ci est de plus en plus marquée par l'hétérogène, ce qui ouvre la voie au questionnement fondamental des concepts de l'identité en tenant compte du caractère performatif des discours identitaires (p. 9-11). Le modèle « postmoderne » autorise une multiplicité d'identités fluides. En d'autres termes, des identités qui non seulement basculent entre les pôles, mais qui les occupent simultanément. Le sexe biologique ne dicte pas nécessairement l'identité d' « homme » ou de «femme », si celle-ci entre en conflit avec la matérialité du corps. Ainsi parle-t-on d'identités genderqueer ou transgenres, ainsi que d'un continuum du genre. Cependant, l'usage des étiquettes 
est problématique puisque les termes sont souvent employés pour englober un groupe important parmi lequel l'éventail des identités s'incarne dans des individus. Mâle/femelle, homme/femme, et masculin/féminin ne sont pas des catégorisations binaires, mais représentent plutôt des échelles mobiles sur lesquelles l'individu se glisse dans un contexte donné. Dans ce modèle, la sexualité est également multiple et mobile, sans présomption d'hétéronorm-ativité. Toutefois, tenant compte de l'ambiguité du terme postmoderne dans le domaine littéraire, et ne voulant pas mêler toutes les conventions du courant postmoderne à l'analyse des textes de Poulin et Laferrière, je propose l'utilisation de transidentitaire. l $^{2}$ Ce terme s'oppose aussi bien aux étiquettes «patriarcal » et «féministe », et permet l'idée d'intégrer la notion québécoise de l' « identitaire ». À cet égard, ce travail examine comment Volkswagen Blues et Le Goût des jeunes filles véhiculent une conception transidentitaire.

\section{L'ÉCRIVAIN DE LA DOUCEUR ET L'ÉCRIVAIN HAÏTIEN-QUÉBÉCOIS-JAPONAIS}

Jacques Poulin, "l'écrivain de la tendresse », dès son premier roman Mon cheval pour un royaume (1967), construit une œuvre qui tourne autour des thématiques intimistes et des figures emblématiques. Les relations homme-femme, l'amour et l'amitié, la survie du français en Amérique, les chats et les mots occupent une place majeure dans ses écrits. Déjà lauréat de nombreux prix littéraires, il reçoit en 2008 le prestigieux prix Gilles-Corbeil, le « Nobel québécois », décerné pour l'ensemble de son œuvre par la Fondation Émile-Nelligan. Selon Robert Lévesque, président du jury :

il y a chez Jacques Poulin le paysage avant le pays, la géographie avant la politique, les odeurs avant les cau-

2. Bien que le problème des étiquettes soit adressé plus haut, et que la définition de trans ${ }^{*}$ permette l'hétérogène, il est toutefois important de ne pas abuser du terme et par là retomber dans l'homogène. Il se peut que le terme risque de gêner les individus et les communautés qui s'identifient comme trans* et qu'il soit donc inapproprié. Il serait peut-être mieux de recourir à un terme plus neutre. 
seurs, et puis et surtout les mots, les mots, ces pierres polies, [...] le poli d'une phrase, au contraire du lustre, le mot infiniment pesé, posé, enlevé, remis à la bonne place, déposé sur la page, le mot avec [...] une balistique de la précision, et en retour, un art de l'émotion retenue, c'està-dire, $[\ldots]$ cette chose qui n'a pas de sexe, ni d'âge, et qui s'appelle la tendresse.

(Montpetit, 2008, para. 12)

Avant de se consacrer à l'écriture, Poulin a fait une formation de conseiller d'orientation et a travaillé dans ce domaine. La psychologie en demeure un élément essentiel de son œuvre puisqu'il cherche toujours « comment ça marche [l'esprit humain] » (Montpetit, 2008, para. 5). Ne se considérant pas un homme public, Poulin reste un écrivain discret, difficile à saisir. Comme les chats qui peuplent son univers romanesque, il demeure énigmatique. Il est difficile de dire si la figure emblématique de l'écrivain désabusé, qui porte souvent le même nom de plume « Jack Waterman », est une représentation de Jacques Poulin.

Bien que certains critiques lui reprochent le caractère répétitif de ses romans, d'autres reconnaissent l'élément essentiel de l'écriture poulinienne : la réécriture. Justement, en parlant de son métier, Poulin dit : " Il y a une condition essentielle pour continuer d'écrire, et c'est de trouver une faiblesse dans le livre que j'ai fait. Et dans le livre suivant, j'essaie de corriger ça » (Desmeules, 2001, para. 5). Selon Biron, Dumont et Nardout-Lafarge (2010), « toute véritable écriture [chez Poulin] est une réécriture, de la même façon que toute véritable lecture est une relecture » (p. 547). Comme Jack, Jacques pose un regard critique sur son œuvre, même si celle-ci lui vaut des honneurs. L'autoreprésentation équivoque et la réécriture sont des thématiques également importantes chez Dany Laferrière.

En 2001, Laferrière a déclaré qu'il était fatigué, «fatigué surtout de [se] faire traiter de tous les noms : écrivain caraïbéen, écrivain ethnique, écrivain d'exil. Jamais écrivain tout court » (Skallerup Bessette, 2013, p. 9). Laferrière a fui la dictature de Jean-Claude Duvalier en 1976 pour Montréal par crainte d'être assassiné en raison de ses 
associations et activités politiques. Le cœur de son œuvre est formé par son «autobiographie américaine», un cycle de dix romans, dont fait partie Le Goût des jeunes filles. Ce touche-à-tout ne veut ni être étiqueté, ni être mis dans des cases : « Je ne sais pas à quel genre appartiennent mes livres. Je les crois hybrides, inclassables [...] » (Sroka, 2010, p. 77). Dans son essai « How to Read Dany Laferrière Without Getting Tired », la critique Lee Skallerup Bessette (2013) suggère que l'action hybride entre le réel et le fictif de son écriture autofictionnel reflète le refus d'être catégorisé et que l'écrivain habite une identité liminale qui « danse aux frontières, toujours en mouvement » (p. 16). Cela dit, il reconnaît ses identités multiples, comme il s'est exclamé lors de son élection à l'Académie française le 12 décembre 2013 : « Ce n'est pas donné à tous d'avoir deux pays. C'est une chance de pouvoir vivre entre le Québec et Haïti » (ICI.Radio-Canada, 2013).

Suscitant la controverse depuis son premier roman, Comment faire l'amour avec un nègre sans se fatiguer (1983), Laferrière, en quelque sorte l'antithèse de Poulin quant à sa participation sur la scène publique, est également difficile à saisir. Bien que la critique féministe ait posé un regard négatif sur les portraits dits « machistes » dans son œuvre, il est tout de même important de considérer les personnages féminins sans recourir à une analyse réductrice. Laferrière joue sur les stéréotypes et confie des fonctions incontournables aux personnages féminins pour construire des textes polyphoniques et complexes. Le contexte historique postcolonial et le Duvaliérisme - caractérisé par les abus de pouvoir et l'exploitation violente du peuple par la milice paramilitaire, les Tontons Macoutes - est essentiel pour une interprétation plus vaste de l'œuvre de Laferrière. Une lecture qui ne tient pas compte de ce contexte risque de repérer uniquement des stéréotypes unidimensionnels, attaqués comme antiféministes.

\section{Présentation des romans : Volkswagen Blues ET LE GOÛT DES JEUNES FILLES}

Le récit principal du Goût des jeunes filles (Montréal : VLB, 2004; désormais GfF) raconte un week-end en 1971. Vieux Os, l'alter ego 
autofictionnel de Laferrière sur lequel repose l'«autobiographie américaine », a quinze ans et se réfugie chez sa voisine Miki parce qu'il se croit la cible des Tontons Macoutes. Marie-Michèle, une adolescente bourgeoise de la riche banlieue Pétionville, s'est infiltrée au groupe de filles qui entoure Miki dans le quartier populaire et elle écrit toutes ses observations dans un journal intime. Vingt ans plus tard, dans le récit qui encadre ce week-end, la publication de ce journal réveille les souvenirs de Vieux Os. Il narre sa version du week-end sous forme cinématographique. Le générique qui commence le récit encadré indique que « l'action se passe à Port-au-Prince, à la fin du mois d'avril 1971. Les années soixante venaient à peine de commencer en Haïti. Avec dix ans de retard. Comme toujours » (GFF, p. 39). Les années soixante représentent « les années de la jeunesse, de cette jeunesse qui se donnait pour mission de tout chambarder, qui remettait tout en question : l'amour, la mort, l'argent, la maternité, la beauté, etc. [...] »(GFF, p. 327). En Haïti, cet esprit n'arrive qu'en 1971 juste avant la mort de François Duvalier, «Papa Doc », et ce week-end représente un moment d'espoir de liberté brièvement vécu avant que le fils du dictateur, Jean-Claude Duvalier, « Baby Doc », arrive au pouvoir.

Volkswagen Blues (Montréal : Babel, 1988 ; désormais VB) s'organise autour d'un voyage, à la fois géographique, temporel et identitaire. Jack Waterman part à la recherche de son frère, Théo, disparu depuis quinze ans. En pleine crise d'écriture, Jack cherche non seulement Théo, mais il est également en quête de sa propre identité. À Gaspé, d'où Théo lui a envoyé la dernière carte postale, Jack rencontre une jeune fille métisse, Pitsémine, qui devient sa compagne de voyage. Surnommée "la Grande Sauterelle », Pitsémine cherche à réconcilier ses identités disparates. Ensemble dans un vieux Volkswagen, ils traversent l'Amérique moderne, l'ancienne Amérique des colons, l'Amérique mythique des héros du Far West, ainsi que l'Amérique des amérindiens. De la Gaspésie à San Francisco, en empruntant les routes anciennes, telles le fleuve Saint Laurent, le Mississippi, la Piste de l'Oregon et la Piste de la Californie, Jack et Pitsémine interrogent l'Histoire ; ils tentent, à travers l'opposition entre passé et présent, entre héros mythiques et pionniers ordinaires, de reconstruire 
l'imaginaire de l'Amérique et de réconcilier l'identitaire québécois et sa complexité grandissante. En effet, ils retrouvent Théo, personnage absent qui représente cet aventurier mythique, atteint d'une creeping paralysis et incapable de reconnaître qui que ce soit. L'évolution de Jack est à l'encontre de celle de son frère : au cours du récit et la quête de l'Ouest, son apprentissage de la vie et de lui-même auprès de Pitsémine lui permet de rentrer à Québec et de recommencer à écrire. Sa quête finie, Jack laisse le Volkswagen à Pitsémine qui n'a toujours pas réconcilié ses identités et qui pourra peut-être les réunir dans la ville ultime de la diversité identitaire, San Francisco.

Selon Boisclair et Saint-Martin (2006), un écrivain « qui donne la parole à un protagoniste du sexe opposé [...] montre un grand intérêt pour l'Autre et une volonté de traverser la frontière des genres » (p. 12), ce qui constitue une manière de véhiculer le transidentitaire. Écrits par deux hommes, les textes mettent en scène un protagoniste accompagné de l'Autre : Laferrière présente deux narrateurspersonnages, un garçon et une fille, tandis que Poulin présente un narrateur extradiégétique qui raconte deux personnages, un homme et une « fille ». Le Goût des jeunes filles, publié pour la première fois en 1992, montre l'intérêt de la part de Laferrière pour l'Autre car il a réécrit le roman en 2004 pour l'augmenter considérablement : MarieMichèle partage la narration et la diégèse également avec Vieux Os. En effet, les femmes sont indispensables au texte et Laferrière le souligne dans l'épigraphe : seules les femmes ont compté pour moi. Une lecture attentive soulève des personnages et des figures nuancés. En donnant la parole aux filles, surtout à Marie-Michelle, Laferrière remet en question l'autorité masculine à ce moment charnière de l'histoire d'Haïti et tente de reconstruire un lieu d'autonomie féminine. Ainsi, les deux écrivains font preuve d'un intérêt pour l'Autre en faisant accompagner leur alter ego, Vieux Os et Jack respectivement, d'un personnage du sexe opposé. Par conséquent, les textes invitent une analyse plus fine du sexe des personnages et la manière dont ils l'expriment. 


\section{LE SEXE}

D’après Boisclair et Saint-Martin (2006), « la désarticulation entre les traits particuliers d'un personnage et son sexe » (p. 12) constitue une manière d'actualiser le modèle " postmoderne », ou en mes termes, d'avancer le transidentitaire, et elles citent la multiplication de l'indifférenciation et de l'indétermination dans l'œuvre de Poulin comme exemple (p. 12). Volkswagen Blues présente deux protagonistes dont les aspects masculins et féminins se brouillent constamment et contribuent à la problématisation identitaire dans le texte. L'incipit raconte la rencontre de Jack et Pitsémine. Celle-ci est présentée comme une jeune fille assez féminine : elle est habillée en robe blanche, les jambes longues et fines, les cheveux longs et tressés. Plus loin, quand Jack se décrit en fonction de son frère, Théo, on nous laisse entendre que Théo est l'idéal masculin et « le contraire de [Jack] : il était grand, un mètre quatre-vingt-dix, les cheveux... noirs comme [Pitsémine] et il ne se cassait pas la tête pour rien » (VB, p. 11). Ainsi, Jack est éloigné du masculin et sa masculinité (traditionnelle) est remise en cause. Selon Edith Vandervoort (2011), il y a un contraste frappant entre Jack et les hommes de la frontière qui se sont battus contre les Indiens et la dure réalité de la Piste de l'Oregon. De plus, les traits de gentillesse, passivité, tristesse, ainsi que son manque d'agressivité et sentiment d'infériorité, sont souvent des traits associés avec les femmes. En dépeignant un homme doux, contemplatif et incertain, Poulin a créé un personnage qui subvertit la patriarchie (p. 317). D’ailleurs, le rapprochement de Théo (l'idéal masculin) et Pitsémine remet en question la féminité (traditionnelle) de celle-ci. En fait, la comparaison commence sa masculinisation. Sa capacité de conduire est particulièrement parlante puisque cette habitude est typiquement réservée aux hommes : « la fille conduisait très bien dans les côtes. Elle n'avait pas besoin de regarder le compteur pour savoir à quel moment elle devait changer les vitesses; elle écoutait simplement le bruit du moteur et ne se trompait jamais » (VB, p. 238). Les personnages occupent des rôles en opposition à leur sexe biologique : Jack cuisine et fait le ménage, tandis que Pitsémine est mécanicienne, un 
métier hautement masculin où Jack avoue son ineptie. L'anecdote des manchots que Pitsémine raconte à Jack illustre et autorise l'inversion des rôles car eux aussi tiennent des rôles inversés : le mâle prend soin de l'œuf, tandis que la femelle part à la pêche. Doucement, Poulin subvertit les rôles traditionnels, et légitime le comportement « masculin » chez les femmes et «féminin » chez les hommes.

Il n'y a rien de doux dans la manière par laquelle Laferrière questionne le lien entre le sexe et les rôles sexués. Le début du Goût des jeunes filles met en scène le modèle féministe à travers tante Raymonde qui questionne l'utilité des hommes : "Les hommes, nous, on n'en a pas besoin. D'ailleurs à quoi ça sert, hein? Qu'est-ce que je ferais d'un homme? » (GFF, p. 16). Elle diminue le rôle des hommes dans leur famille : "Aucun homme ne peut nous séparer, il y a eu un seul homme dans cette famille et c'est ton père, le seul... Je sais que Gilberte a eu des amants, et Ninine aussi quelquefois, mais c'était toujours passager, ce n'était pas vraiment important... » (GFF, p. 19). Pour tante Raymonde, le rôle d'un homme est purement physique, soit pour les travaux manuels, soit pour combler les envies sexuelles et les besoins de la reproduction. L'homme est ainsi réduit à son corps, voire son sexe biologique, ce qui maintient une vision binaire du sexe puisque tante Raymonde ne propose pas que les femmes remplissent ces fonctions. En revanche, elle s'occupe des besoins matériels de toute la famille. Le rôle masculin de soutien de famille peut être subverti par une femme, mais en gardant l'aspect féminin.

Cela dit, la masculinité est remise en question par Vieux Os qui ne se conforme pas aux normes sociales traditionnelles relatives aux rôles des hommes telles que véhiculées par le roman où les personnages masculins n'ont souvent que des rôles violents et cruels (Papa, Frank et les Tontons Macoutes) ou absent (son père). Bien qu'il soit jaloux de la liberté qu'il perçoit chez son ami Gégé qui incarne cette masculinité traditionnelle, Vieux Os est un garçon doux et sensible qui lit de la poésie. En famille, Vieux Os prend l'aspect masculin puisqu'il est le seul mâle, mais par rapport à Gégé, il paraît moins masculin. Encerclé par les filles chez Miki, il retient le statut de "garçon », mais sa masculinité est remise en question puisque il lui manque les 
traits typiquement masculins, surtout l'agressivité.

Dans Volkswagen Blues, bien que Pitsémine effectue des actes typiquement féminins, comme se brosser les cheveux et porter des robes, elle alterne la stylisation féminine et masculine de son corps. Juste avant de se mettre en drag à l'hôtel YMCA, Pitsémine s'hyper-féminise en se mettant nue devant Jack. Mais en drag, Pitsémine ne perd pas son allure : Jack la trouve « très drôle [et son] chapeau de tennis est absolument irrésistible » $(V B$, p. 112). En le remerciant elle dit, «[...] je suis votre homme! » $(V B$, p. 112), brouillant davantage son l'identité sexuelle. Plus loin, Saul Bellows, l'écrivain anglais et lauréat du prix Nobel qui reconnait que Pitsémine est une fille, dit : «When you're looking for your brother, you're looking for everybody !» (VB, p. 119). Un tel énoncé laisse entendre que la masculinité et la féminité sont des caractéristiques qui ne sont pas incompatibles, mais, comme l'idée d'un continuum de genre le soutient, peuvent empiéter l'une sur l'autre. Ceci rejoint les propos de Boisclair et Saint-Martin (2006) qui disent que « la non-considération de la spécificité sexuelle peut mener à tenir le féminin pour humain, pour universel » (p. 11). Ainsi, le masculin et le féminin peuvent se retrouver chez tout individu à des degrés divers. Poulin cherche à soulever les ressemblances de Jack et Pitsémine : ils parlent «d'une seule voix » $(V B$, p. 207), ils marchent « côte à côte » $(V B$, p. 218), ils sont « deux vrais zouaves!» $(V B, \mathrm{p}$. 260 ) et «les deux plus grands menteurs de l'Amérique du Nord! » $(V B$, p. 84). Le texte insiste sur une rencontre égale entre l'homme et la fille. «Chacun à leur façon, l'homme et la fille étaient maniaques des musées » (VB, p. 134, je souligne). Jack et Pitsémine peuvent avoir des identités similaires, soit masculin et/ou féminin, et les exprimer chacun à leur façon. Par conséquent, ce type d'énoncé illustre le transidentitaire qui insiste sur la pluralité et l'individualité du genre.

Dans Le Goût des jeunes filles, le sexe et le corps sont fortement liés. Selon Marie-Michèle, le rôle maternel est indissociable du corps féminin et son questionnement du maternel est essentiel à la critique qu'elle fait de sa mère. Elle rêve d'avoir été élevée comme les enfants de la classe populaire qu'elle voit dans les rues en allant à l'école :

Ces mendiantes que je vois toujours avec un bébé atta- 
ché à leurs hanches, la bouche suçant frénétiquement un sein flasque où ne semble plus rester une seule goute de lait. D’abord, ma mère n'aurait jamais accepté que je maltraite à ce point son sein (elle y tient), elle ne m'aurait non plus jamais nourrie de ses dernières réserves, comme le font, avec abnégation, toutes ces mères. (GFF, p. 47)

Bien que le jugement négatif sur la capacité maternelle de sa mère soit lié au déni du sein, et semble en soit une réduction au corps, le rôle de mère n'est pas présenté comme inéluctable. Les traditions littéraires québécoises, surtout les romans du terroir, enfermaient les personnages féminins dans le rôle traditionnel de la maternité; devenir mère était une fin obligée, voire indiscutable. Marie-Michèle, elle, ne veut pas avoir d'enfants, mais ce rejet de la fonction reproductive ne fait planer aucun doute sur son sexe, ni son identité sexuelle. Le texte rejette la notion d'un lien obligatoire entre les femmes, la féminité et la maternité, et ainsi remet en cause un des fondements de la conception de ce qu'est une femme.

\section{LE GENRE}

L'ambigüité identitaire, surtout la fluidité de genre, sont centrales aux textes et se manifestent à travers les représentations d'entre-deux que sont les identités plurielles et le mouvement. Selon Pierre Hébert, l'un des thèmes essentiels chez Jacques Poulin est l'équilibration intérieure des parties féminine et masculine de notre être (1997, p. 8). Comme le dit Pitsémine : «Je suis partagée entre les deux, et je sais que ça va durer toujours » (VB, p. 59). Poulin crée un entre-deux constamment partagé où le transidentitaire est autorisé. Jack et Pitsémine cherchent à réconcilier leurs propres identités, mais il y a aussi une tentative de parcourir la distance entre celles-ci. Les postes de traite représentent l'entre-deux où l'exploration est valorisé : « [...] les postes de traite étaient situés le long des cours d'eau... alors ils n'ont pas seulement été utilisés pour la traite des fourrures : on s'en est servi aussi comme points de départ pour toutes sortes d'expédi- 
tions » $(V B$, p. 45). Aucun côté n'est privilégié, mais l'importance est accordée au milieu.

Les postes de traite et les points de départ sont aussi importants car c'est aux endroits d'échanges qu'ils reçoivent des informations pour le prochain bout de voyage. De cette manière, le texte et les personnages sont rarement immobiles, mais constamment en transit. Par exemple, en passant la ville de Brantford, elle veut passer la nuit dans le cimetière où est enterré le chef Thayendanegea (Joseph Brant) pour « se réconcilier avec elle-même » $(V B$, p. 87). Dans ce contexte particulier, Pitsémine se dessaisit du drag et se reféminise. Devant un ancien chef Mohawk, peuple qui faisait partie de la confédération des Six-Nations dans laquelle les femmes ont joué un rôle important, " elle avait l'air de croire que, d'une façon ou d'une autre, le vieux chef pouvait l'aider à se connaître elle-même » $(V B$, p. 88$)$. Ainsi, elle enlève son costume de garçon et remet une robe pour reprendre le sexe et le genre féminin. Malgré ceci, le résultat est un échec : « il ne s'est rien passé du tout » $(V B$, p. 93). La réconciliation ne peut se faire qu'avec la mise en équilibre de toutes ses parties. Ce n'est qu'à San Francisco, la ville ultime de la diversité et de subversion des normes sociales, qu'elle peut s'établir : « elle pensait que cette ville, où les races semblaient vivre en harmonie, était un bon endroit pour essayer de faire l'unité et de se réconcilier avec elle-même » (VB, pp. 317-318). Jadis, l'entre-deux n'était pas permis dans les sociétés. En tentant de réconcilier l'Histoire de l'Amérique du Nord dans son intégralité en joignant le passé au présent, Jack et Pitsémine deviennent une nouvelle représentation d'un couple originel; il n'y a plus de guerre entre les sexes, ils ne sont pas en compétition et ils s'entraident le long du roman. Quand Jack demande pourquoi elle s'occupe de lui, Pitsémine explique : «parce que... on est deux. On est ensemble. On ne peut pas vivre comme si on était séparés » $(V B, p .147)$. Le texte souligne leur identité plurielle, à la fois leur statut en tant que deux individus, et leur statut commun en tant que couple. Plus loin, Jack dit que Théo « est à moitié vrai et à moitié inventé. Et s'il avait une autre moitié... [...] La troisième moitié serait moi-même » $(V B$, p. 149). Cette troisième moitié remet en question le binarisme et met en valeur l'hétérogène. 
L'entre-deux où le masculin et le féminin coexistent fait preuve d'un lieu de coopération, illustré lors d'une panne ; ils travaillent ensemble pour résoudre le problème, tout en subvertissant les rôles :

Comme elle travaillait au soleil, il lui fit une sorte d'auvent en utilisant la couverture de flanelle. Pour qu'elle ne fût pas obligée de rester en position accroupie, il lui apporta le petit tabouret en vinyle jaune du Volks. Il avait versé de l'eau fraîche dans un grand plat et, de temps en temps, il lui épongeait le front avec une serviette mouillée [...]. Et lorsque tout fut terminé et que le moteur [...] se mit à tourner comme un neuf, il [...] déclara à la fille, qui avait la figure barbouillée d'huile et les mains et les bras noircis jusqu'au coude : «Vous êtes le plus beau mécanicien que j'aie jamais vu! Je vous adore! » Il se laissa aller à toutes sortes d'excès de langage » (VB, p. 267-68, je souligne).

La coexistence du masculin et féminin est représentée non seulement pas la coopération entre Jack et Pitsémine, mais par le narrateur qui permet la concurrence des genres. Le narrateur maintient le sexe femelle de Pitsémine et le sexe mâle de Jack en les appelant invariablement « la fille » et «l'homme » respectivement, sans exiger qu'ils renoncent leurs identités de genre fluides ou à leurs rôles nontraditionnels. Pitsémine est à la fois « fille » et « le plus beau mécanicien ». Cette concurrence du masculin et du féminin s'inscrit dans le transidentitaire qui insiste sur la pluralité.

La pluralité identitaire se retrouve également dans Le Goût des jeunes filles sur le plan structurel. Le texte comporte des formes multiples entremêlées - littéraires, cinématographiques, poétiques et journalistiques -, et souligne un refus des conventions traditionnelles du romanesque. Ainsi le texte fonctionne comme une revendication du pouvoir de l'écrivain. La multiplicité des formes recèle l'hétérogène, exigeant un mode de lecture hybride chez les lecteurs. Le récit encadré des souvenirs de Vieux Os prend la forme d'un scénario «Weekend à Port-au-Prince (un film écrit, filmé et réalisé par Dany Laferrière) » et la voix narrative de Marie-Michèle vient se mêler en forme de 
journal intime. Ces entrées n'appartiennent pas strictement au même week-end puisqu'elles sont publiées au présent ( 25 ans après le weekend), et la fin du texte est un entretien avec Marie-Michèle à propos de son journal. Laferrière refuse de se laisser encadrer dans des catégories romanesques traditionnelles. Dans ce texte, il y a une liberté de forme, loin de la forme classique de l'autobiographie et de la fiction. De plus, la mise en scène d'une narratrice permet un point de vue féminin. Toutefois, l'entre-deux ne produit pas le même effet de fluidité chez les personnages.

Vieux Os et Marie-Michèle se rencontrent à la fois dans le texte et dans la maison de Miki : «C'est incroyable combien on était à la fois si proche et si loin : pendant que j'enregistrais dans ma tête et dans mon cœur ce qui se passait de l'autre côté de la rue, durant ce fameux week-end, eh bien, au même moment, elle notait presque les mêmes évènements dans son journal » (GFF, p. 33). Bien que la maison de Miki soit un lieu de rencontre, l'entre-deux n'est que possible à travers l'écriture et la lecture du texte, contrairement au cas de l'union entre Jack et Pitsémine. Ici, les personnages ne peuvent pas s'intégrer l'un à l'autre : "C'est l'époque qui est ainsi tricotée : on sait ce qu'on fait, on sait qui on est, pourtant on ignore ce que fait l'autre au même moment, bien que l'on soit tous les deux sur la même longueur d'onde » (GFF, p. 33). Alors que Poulin rapproche ses personnages, les personnages laferriens gravitent dans une orbite à la fois proche et éloignée. Cela dit, le texte, et surtout la réécriture, insistent sur la présence des deux points de vue : «[...] pour comprendre bien cette époque, il sera nécessaire de regarder mon petit film [...] tout en lisant le journal de Marie-Michèle. Si mon Eil objectif balaie la surface des choses, l'Oreille ultrasensible de Marie-Michèle descend jusque dans les grandes profondeurs pour capter certaines vibrations » (GFF, p. 33). Pourtant, en tant que personnages, ils ne peuvent pas partager ce lieu de rencontre et ils racontent leurs observations en alternance. C'est seulement à travers le texte qu'ils peuvent, vingt-cinq ans plus tard, coexister, l'un à côté de l'autre, en égalité. Ainsi, le texte devient un lieu de concurrence masculine et féminine, tout en se différenciant l'un de l'autre. 
Par la voix de Marie-Michèle, Laferrière légitime le questionnement de la condition des femmes et l'état du féminisme à l'époque de Papa Doc. Cependant, le discours féministe de Marie-Michèle est lacunaire. Elle pense que les filles chez Miki vivent plus librement, mais en fait le mode de vie des filles est imposé par des injustices sociales, qu'elles en soient conscientes ou non. Marie-Michèle reproche à sa mère et aux femmes de sa classe d'être hypocrites et elle les incite à s'émanciper de leur prison dorée, mais les problèmes existent au niveau de la structure sociale «basée sur trois choses fondamentales : la richesse familiale, l'exploitation du peuple et la corruption de la classe politique en fonction » (GFF, p. 80). Ainsi, la société et surtout le développement du féminisme est entravé par ces structures défendues par la mère de Marie-Michèle qui représente l'élite minoritaire. L'hypocrisie et la stagnation du cercle doré contrastent fortement avec le cercle de Miki « où les liens de sang n'existent pas, rien n'est permanent. Tout bouge constamment » (GFF, p. 84). Enfin, Marie-Michèle rejette irrévocablement le Cercle et s'installe à New York où elle se libérera des contraintes des structures sociales traditionnelles.

Le mouvement dit beaucoup sur l'identité dans les deux textes. Pitsémine, née dans une roulotte, reste en mouvement le long du texte et même après la fin de celui-ci puisque Jack lui donne le Volks. Justement, la fluidité, ou la traversée des « frontières » identitaires, devient l'identité elle-même, ce qui rejoint le propos de Judith Butler (1990) qui soutient dans son ouvrage Trouble dans le genre qu'il est impossible de décrire un individu transsexuel par « homme » ou «femme », qu'il faut « témoigner de la constante transformation - transformation qui « est » la nouvelle identité -, et même de l' « entre-deux » qui interroge ce qu'est une identité genrée » (p. 32). Comme le Volks, Pitsémine représente une identité fluide qui n'arrête pas d'explorer. Cela dit, le parcours est également important chez Jack, mais se manifeste dans l'écriture. Dans leur dernière conversation, Pitsémine lui rappelle que «l'écriture est une forme d'exploration » (VB, p. 318). L'écriture, comme le voyage, permet d'explorer l'identité et de franchir, voire d'occuper, les frontières de l'entre-deux. Leur adieu à l'aéroport évoque une telle occupation partagée de l'entre-deux à la fois 
du corps et de l'esprit :

Alors ils se serrèrent l'un contre l'autre, assis au bord de leur siège, les genoux mêlés, et ils restèrent un long moment immobiles, étroitement enlacés comme s'ils n'étaient plus qu'une seule personne.

(VB, p. 320)

Selon Pierre L’Hérault (1989), « les rapports entre Jack et Pitsémine sont un jeu de déplacement à l'intérieur d'un champ identitaire d'où le monologique est exclu. Jack n'est jamais Pitsémine ; pourtant il l'est. De même Pitsémine n'est jamais Jack; pourtant elle l'est de quelque façon [...] Dans ce jeu [...] le personnage se déplace, non seulement par rapport à l'autre, mais aussi par rapport à lui-même, dans un mouvement de décentrement qui va de l'unique au multiple » (p. 34). Vu sous cet angle, il est évident que le texte fait ressortir les valeurs du transidentitaire.

\section{LA SEXUALITÉ}

En ce qui concerne la sexualité, Jack exprime succinctement les valeurs de liberté et de respect : «Dans le domaine sexuel, je trouve qu'on devrait avoir le droit de tout faire à condition de respecter la liberté de l'autre » $(V B$, p. 82). Jack applique cette philosophie lorsqu'à Saint Louis Pitsémine sort avec un autre homme. Quand elle rentre très tard une nuit, Jack ne veut pas être hypocrite et lui faire la morale : « Je n'aime pas la morale. Vous êtes libre et vous n'êtes pas à moi » (VB, p. 138). Leur relation est fondée sur le respect mutuel et l'égalité, et les tentatives sexuelles entre eux tombent à l'eau lorsqu'il y a une inégalité de pouvoir. Contrairement, la sexualité dans Le Goût des jeunes filles est toujours en rapport avec le pouvoir et deviennent une stratégie de survie. Choupette exprime le même principe de liberté que Jack dans Volkswagen Blues, bien que plus crûment, quand elle parle de sa relation avec Papa : « [...] C'est pas moi qui le retiens... $\mathrm{Si}$ ça ne fait pas son affaire, il sait toujours quoi faire... Je l'ai averti : " $\mathrm{OK}$, tu veux rester, reste, mais mon cul est à moi, et à personne d'autre. » Et il était d'accord » (GFF, p. 198). Dans le texte, les rôles 
masculins et féminins sont souvent inversés : les filles détiennent le pouvoir sexuel, tandis que les hommes sont soumis. Frank, l'amant de Pasqualine, est aussi un homme-objet, mais il représente explicitement le pouvoir exploiteur et le danger auquel les filles font face en fréquentant les Tontons Macoutes puisqu'il travaille actuellement à Fort Dimanche, la prison où les Tontons Macoutes torturent les détenus. Bien que Frank soit un tueur, la conversation entre Pasqualine et Marie Erna montre l'inversion du pouvoir :

- Comment fais-tu avec lui ?

- Je l'ai dompté.

- C'est tout?

- C'est tout.

- Comment fais-tu alors pour baiser avec un tueur ?[...]

- Ce n'est pas différent d'avec un autre, sauf que quelqu'un comme lui est plus sensible, tu vois... Comme si tu manipulais une bombe.

- C'est dangereux!

- Très [...] Et puis, quand il jouit, il appelle sa maman.

[...] Un vrai bébé...

(GfF, p. 207)

Cela dit, il n'y a pas tout à fait un renouvellement de la conception du sexe/genre car l'univers fictionnel, ancré dans le contexte historique d'Haïti en 1971, insiste sur un lien étroit entre la sexualité et le pouvoir. Dans le cas de Pasqualine, elle est obligée de s'engager dans une relation sexuelle avec Frank pour sauver son frère qui a été emprisonné. De même, Miki et Choupette participent à des relations où la sexualité est une obligation contractuelle, qu'elle soit sous-entendue ou explicite. Les relations sans intimité, sans sentiment, qui sont des relations de pouvoir, transforment l'être humain en objet. Ainsi, le discours féministe est renversé sur lui-même à partir du moment où l'on fait de l'Autre un objet. L'humain est évacué du sexe : les figures d'hommes sont abusives, sans émotions, tandis que les filles, bien qu'elles aient des émotions, sont également abusives. Laferrière dit que « c'est la sexualité comme instrument de pouvoir politique, de pouvoir social, de pouvoir économique [...] et on a ceux qui sont 
prêts à vendre la seule chose qu'ils ont, c'est-à-dire leur jeunesse et leur corps » (Sroka, 2010, p. 76). Le texte critique une société encore en guerre - les hommes contre les femmes, les pauvres contre les riches. Ces liaisons « dangereuses » (le roman de Choderlos de Laclos est abondamment cité dans le journal de Marie-Michèle) détruisent la vie humaine, et pour les filles démunies, ce ne sont pas des jeux de société, mais de survie.

Dans Volkswagen Blues, Jack et Pitsémine partagent bien de moments de complicité et leurs gestes affectueux pourraient être interprétés comme des avances sexuelles. Toutefois, le texte ne repose pas sur la présomption d'hétérosexualité. Les gestes n'aboutissent pas à des scènes de passion typiques d'un couple hétérosexuel, mais créent une dynamique douce et tendre. Ce n'est pas une relation de pouvoir, mais de complicité et de rencontre. Ce qui les excite véritablement, c'est leur parcours. Par exemple, à Saint Louis en vue du Gateway Arch, « tout de suite, Jack et la fille avaient été séduits par l'arc métallique dont la silhouette très délicate [...] »(VB, p. 133), et Pitsémine affirme la corrélation entre le mouvement et le bonheur : «quand on est sur la route, je suis contente » (VB, p. 179). Le mouvement, le parcours du territoire, de l'identité et de l'écriture sont plus séduisants, bien qu'ils se témoignent beaucoup d'affection, voire amour, l'un pour l'autre. D'ailleurs, les frontières sont d'autant plus importantes dans le récit, et, particulièrement, leur franchissement. Quand les personnages franchissent une frontière, c'est un évènement qui dénote un changement ou une transformation importante. Les ponts, les routes, les croisements, les intersections, ce texte leur attribue une qualité transformative. Le geste de traverser, non seulement le parcours géographique, mais le parcours intérieur, est essentiel pour la quête identitaire et s'inscrit dans le transidentitaire. Jack dit, « Je voudrais qu'ils me disent ce qu'ils aperçoivent de l'autre côté et s'ils ont trouvé comment on fait pour traverser » (VB, p. 131). C'est seulement en traversant qu'il fera sa propre réconciliation.

C'est à la ligne de partage des eaux - où les eaux se divisent et choisissent de couler vers un côté ou l'autre - que Pitsémine propose de faire l'amour. Bien que ce soit un acte hétérosexuel entre un 
homme et une femme, les rôles sont inversés. Pitsémine est « l'agresseur » et elle prend le contrôle, ce qui est typiquement masculin. Jack prend le rôle de soumission qui est typiquement féminin. Non seulement l'inversement subvertit les rôles de genre, mais l'inégalité du pouvoir fait de l'acte une faillite. Jack est incapable de prendre parole pour prévenir Pitsémine, et il jouit prématurément. Typique du discours du féministe, Pitsémine est insatisfaite par l'acte. Selon Edith Vandervoort (2011), Jack est une figure émasculée qui est incapable d'accomplir le comportement essentiel chez tous les animaux : la procréation. L'échec de l'acte sexuel sur la ligne de partage est symbolique de son incapacité de réaliser son rôle d'homme. Elle avance une raison symbolique, que Jack et Pitsémine sont plus comme frère et sœur et qu'une telle union serait incestueuse (p. 321). Or, une conception transidentitaire permet une lecture plus nuancée du sexe et des genres. Selon moi, plutôt qu'une relation fraternelle, il s'agit d'une relation d'amour où les participants ont un comportement sexuel non-traditionnel, c'est-à-dire sans sexe. Comme la philosophie exprimée par Jack au début, le texte prône l'égalité et, qu'importe les rôles, une relation déséquilibrée est vouée à l'échec. La symbolique de faire l'amour directement sur la ligne de partage - le point même sur lequel les eaux basculent avant de choisir un côté - indique une tentative de célébrer la rencontre des sexes. En fait, il illustre davantage l'entre-deux et le basculement possible, tout comme les eaux qui glissent tantôt vers le Pacifique, tantôt vers l'Atlantique. Plus loin pendant la nuit, Pitsémine dit « qu'elle n' [est] ni une Indienne, ni une Blanche, qu'elle [est] quelque chose entre les deux et que, finalement, elle n' [est] rien du tout » (VB, p. 246), mais Jack marque son désaccord en disant : " Je trouve que vous êtes quelque chose de neuf, quelque chose qui commence. Vous êtes quelque chose qui ne s'est encore jamais vu » (VB, p. 247). L'entre-deux devient un espace de création, où tout est possible comme le transidentitaire nous ferait entendre. 


\section{CONCLUSION}

De nos jours, bien que la politique antigaie et les mouvements anti-homosexuels ne soient pas acceptés au Canada, il existe toujours de la confusion, des malentendus, de l'hostilité, voire de la violence, envers les individus et les communautés trans*. Malgré la place importante du transidentitaire dans le dialogue qui se déroule autour du genre, les nouveautés ne sont pas toujours accueillies chaleureusement et après leur conversation, Jack « rêva que la Grande Sauterelle était une extra-terrestre » $(V B$, p. 247). La conception binaire du sexe et du genre traditionnel, soutenue par un langage inadéquat pour nommer l'ambiguïté de l'entre-deux, contribue à cette image « extraterrestre ». Autant accepter notre diversité et remettre en questions les binarités strictes telles que le masculin et le féminin qui occultent le transidentitaire. La littérature peut être un puissant moteur de développement des identités, et il nous incombe en tant que critiques et chercheurs de participer à celui-ci. Comme le dit Sherry Simon (1991) : « la critique contribue, au même titre que les fictions qu'elle commente, à donner existence aux espaces identitaires qu'elle nomme » (p. 11). Volkswagen Blues de Jacques Poulin et Le Goût des jeunes filles de Dany Laferrière sont des textes qui participent à ce renouvellement transidentitaire. Ce sont des textes qui dépassent la binarité stricte du sexe, du genre et de la sexualité, et ouvrent la voie vers une plus grande acceptation des ambigüités identitaires, voire vers le développement du transidentitaire dans la littérature québécoise. 


\section{RÉFÉRENCES}

Biron, M., Dumont, F., \& Nardout-Lafarge, E. (2010). Histoire de la littérature québécoise. Montréal : Boréal.

Boisclair, I. (2005). Le jeu du genre : Évolution des postures critiques initiées par le féminisme. Québec français, 137, 35-38.

Boisclair, I. (2008). Le personnage intersexué : Voie de renouvellement de l'imaginaire des sexes/genres? Nouvelles Questions Féministes, 27(1), 63-78. http://dx.doi.org/10.3917/nqf.271.0063

Boisclair, I., \& Saint-Martin, L. (2006). Les conceptions de l'identité sexuelle, le postmodernisme et les textes littéraires. Recherches Féministes, 19(2), 5-27. http://dx.doi.org/10.7202/014841ar

Boisclair, I., \& Saint-Martin, L. (2009). Masculin/féminin chez les romanciers québécois contemporains : L'idée de différence entre maintien et renouvellement. Contemporary French and Francophone Studies, 13(1), 45-54. http://dx.doi.org/10.1080/17409290802606812

Bordeleau, F. (1993). Un adolescent en Haïti. Lettres québécoises: La revue de l'actualité littéraire, 70, 21-21.

Butler, J. (1988). Performative Acts and Gender Constitution : An Essay in Phenomenology and Feminist Theory. Theatre fournal, 40(4), 519-531. http://dx.doi.org/10.2307/3207893

Butler, J. (2005). Trouble dans le genre : Le féminisme et la subversion de l'identité [1990]. (C. Kraus, Trans.). Paris : La Découverte.

Coleman, D. (1998). Masculine Migrations : Reading the postcolonial male in "New Canadian" narratives. Toronto : University of Toronto Press.

Desmeules, C. (2011). Jacques Poulin, l'homme et ses doubles. Le Devoir, accédé 15 mars 2014, http ://www.ledevoir.com/culture/livres/ 332646/jacques-poulin-l-homme-et-ses-doubles

Gilbert, P. R., \& Dufault, R. L. (Eds.). (2001). Doing gender : FrancoCanadian women writers of the 1990s. Madison [N.J.]; London; Cranbury, NJ : Fairleigh Dickinson University Press; Associated University Presses.

Gopalan, K. (2008). (Re)Presentations of the Female Body in the Works of Canadian Women Writers. New Delhi : Prestige Books. 
Gould, K. (1990). Writing in the Feminine : Feminism and Experimental Writing in Quebec. Carbondale; Edwardsville : Southern Illinois University Press.

Hébert, P. (1997). Jacques Poulin : la création d'un espace amoureux. University of Ottawa Press.

Hopwood, S. (2011). Subverting the Dictatorship in Dany Laferrière's Le Goût des jeunes filles. French Review : fournal of the American Association of Teachers of French, 84(3), 474. ICI.Radio-Canada.ca. (n.d.). Dany Laferrière entre à l'Académie française | ICI. RadioCanada.ca. Retrieved March 10, 2014, from http ://ici.radio-canada. ca/nouvelles/arts_et_spectacles/2013/12/12/004-dany-laferriereacademie-francaise.shtml

Joubert, L., \& Boisclair, I. (Eds.). (2000). Trajectoires au féminin dans la littérature québécoise (1960-1990). Québec, Québec : Editions Nota bene.

L'Hérault, P. (1989). Volkswagon Blues : traverser les identités. Voix et Images, 15(43), 28-42. http://dx.doi.org/10.7202/200813ar

Laferrière, D. (2004). Le goût des jeunes filles. Montréal : VLB éditeur.

MacFarlane, H. (2009). Volkswagen Blues twenty-five years later : Revisiting Poulin's Pitsemine. Studies in Canadian Literature, 34(2), 6.

Mathis-Moser, U. (2003). Dany Laferrière : La dérive américaine. Montréal : vlb éditeur.

Montpetit, C. (2008). Le «Nobel Québécois» à Jacques Poulin. Le Devoir, consulté le 19 février 2014, http://www.ledevoir.com/culture/ livres/214065/litterature-le-nobel-quebecois-a-jacques-poulin

Paterson, J. M. (1994). Ni l'un, ni l'autre : L'Ambivalence du discours de l'hétérogène dans Volkswagen Blues. University of Toronto Quar-

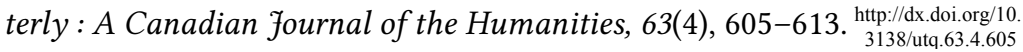

Poulin, J. (1988). Volkswagon Blues. Montréal : Babel.

Royer, J. (1989). Le Devoir - Google News Archive Search. Retrieved February 23, 2014, from http ://news.google.com/newspapers ?id= mwQyAAAAIBAJ\&sjid=BeIFAAAAIBAJ\&pg $=1506 \% 2 \mathrm{C} 3475069$

Simon, S., L'Hérault, P., Schwartwald, R., \& Nouss, A. (1991). Fictions de l'identitatire au Québec. Montréal : XYZ éditeur. 
Skallerup Bessette, L. (Ed.). (2013). Dany Laferrière : Essays on his works. Toronto : Guernica Editions.

Sroka, G. (2010). Conversations avec Dany Laferrière. Montréal : Editions de la Parole Métèque. Trans*. (n.d.). GATE - Global Action for Trans* Equality. Retrieved from http ://transactivists.org/trans/ Vandervoort, E. B. (2011). Subverting the Masculine Image in Jacques Godbout's Salut Galarneau and Jacques Poulin's Volkswagen Blues. In Masculinities in Twentieth- and Twenty-First Century French and Francophone Literature (pp. 305-324). Newcastle upon Tyne : Cambridge Scholars Publishing. 